\title{
Supplement - Introducing PebbleCounts: A grain-sizing tool for photo surveys of dynamic gravel-bed rivers
}

Benjamin Purinton ${ }^{1}$ and Bodo Bookhagen ${ }^{1}$

${ }^{1}$ Institute of Earth and Environmental Science, Universität Potsdam, Potsdam, Germany

Correspondence: Ben Purinton (purinton@uni-potsdam.de)

S1. Command-line Variables for PebbleCounts

Table S1 shows the command-line variables for Pebble Counts (KMS approach) and Table S2 shows the command-line variables for PebbleCountsAuto (AIF approach). 
Table S1. Command-line variable flags in PebbleCounts and their meaning. The default values are effective for most images.

\begin{tabular}{|c|c|c|}
\hline Variable Flag & Meaning (units) & Default Value(s) and Suggested Range \\
\hline im & Image to run, including path to folder & No default \\
\hline ortho & Georeferenced orthoimagery flag & No default, ' $y$ ' for orthoimagery, 'n' for top-down \\
\hline input_resolution & Input resolution if not orthoimage $(\mathrm{mm})$ & No default, calculate from eq. (3) \\
\hline subset & Interactively subset image & Default no ('n') \\
\hline sand_mask* & $\begin{array}{l}\text { Name, including path, to a sand mask if one al- } \\
\text { ready exists }\end{array}$ & No default \\
\hline otsu_threshold* & $\begin{array}{l}\text { Percentage of Otsu value to threshold shadows } \\
\text { by (percentage of 100) }\end{array}$ & No default, suggested value of 50 \\
\hline $\max G S^{*}$ & Expected maximum a-axis grain size $(\mathrm{m})$ & Default 0.3 \\
\hline cutoff* & Minimum b-axis length to be counted (pixels) & Default 20 , can be raised \\
\hline min_sz_factors $*$ & $\begin{array}{l}\text { Factors to multiply cutoff at each scale, used to } \\
\text { cleanup masks for easier clicking }\end{array}$ & $\begin{array}{l}\text { Default }[50,5,1] \text { for three scales (large to small) for } \\
\sim 1 \mathrm{~mm} / \text { pixel imagery, double for }<0.8 \mathrm{~mm} / \text { pixel }\end{array}$ \\
\hline win_sz_factors* & Factors to multiply $\max G S$ by at each scale & $\begin{array}{l}\text { Default }[10,3,2] \text { for three scales (large to small), can } \\
\text { be changed } \pm 0.5-1.5 \text { to get more or less windows }\end{array}$ \\
\hline improvement_ths* & $\begin{array}{l}\text { Improvement threshold values that tell k-means } \\
\text { when to halt (fraction of } 1 \text { ) }\end{array}$ & $\begin{array}{l}\text { Default }[0.01,0.1,0.1] \text { for three scales (large to } \\
\text { small), can be varied from } 0.01-0.2\end{array}$ \\
\hline coordinate_scales* & Fraction to scale $x, y$ coordinates (fraction of 1 ) & $\begin{array}{l}\text { Default }[0.5,0.5,0.5] \text { for three scales (large to } \\
\text { small), can be varied from } 0.3-0.7\end{array}$ \\
\hline overlaps* & $\begin{array}{l}\text { Fraction of overlap between windows (fraction } \\
\text { of } 1 \text { ) }\end{array}$ & $\begin{array}{l}\text { Default }[0.5,0.3,0.1] \text { for three scales (large to } \\
\text { small), can be varied from } 0-0.5 \text { at each scale }\end{array}$ \\
\hline first_nl_denoise* & Strength of first non-local means denoising & Default 5 , can be varied \pm 1 \\
\hline nl_means_chroma_filts* & $\begin{array}{l}\text { Strength of windowed non-local means denois- } \\
\text { ing }\end{array}$ & $\begin{array}{l}\text { Default }[3,2,1] \text { for three scales (large to small), can } \\
\text { be varied } \pm 1\end{array}$ \\
\hline bilat_filt_szs* & Size of bilateral filtering windows (pixels) & $\begin{array}{l}\text { Default }[9,5,3] \text { for three scales (large to small), can } \\
\text { be varied from 3-9 }\end{array}$ \\
\hline tophat_th* & $\begin{array}{l}\text { Upper percentile threshold to take from top-hat } \\
\text { filter for edge detection (fraction of } 1 \text { ) }\end{array}$ & Default 0.9 , can be varied from $0.8-0.95$ \\
\hline sobel_th* & $\begin{array}{l}\text { Upper percentile threshold to take from sobel fil- } \\
\text { ter for edge detection (fraction of 1) }\end{array}$ & Default 0.9 , can be varied from $0.8-0.95$ \\
\hline canny_sig* & Canny filtering sigma value for edge detection & Default 2 , can be varied from $1-2$ \\
\hline resize & Value to resize windows by (fraction of 1 ) & $\begin{array}{l}\text { Default } 0.8 \text {, can be varied from } 0.5-0.99 \text { if you want } \\
\text { a smaller }(0.5) \text { or larger }(0.99) \text { pop-up window }\end{array}$ \\
\hline
\end{tabular}

*Influence on results 
Table S2. Command-line variable flags in PebbleCountsAuto and their meaning. The default values are effective for most images.

\begin{tabular}{|c|c|c|}
\hline Variable Flag & Meaning (units) & Default Value(s) and Suggested Range \\
\hline$i m$ & Image to run, including path to folder & No default \\
\hline ortho & Georeferenced orthoimagery flag & No default, 'y' for orthoimagery, 'n' for top-down \\
\hline input_resolution & Input resolution if not orthoimage (mm) & No default, calculate from eq. (3) \\
\hline subset & Interactively subset image & Default no ('n') \\
\hline sand_mask* & $\begin{array}{l}\text { Name, including path, to a sand mask if one al- } \\
\text { ready exists }\end{array}$ & No default \\
\hline otsu_threshold* & $\begin{array}{l}\text { Percentage of Otsu value to threshold shadows } \\
\text { by (percentage of 100) }\end{array}$ & No default, suggested value of 50 \\
\hline cutoff* & Minimum b-axis length to be counted (pixels) & Default 20 , can be raised \\
\hline percent_overlap* & $\begin{array}{l}\text { Maximum allowable overalp between neighbor- } \\
\text { ing ellipses for filtering suspect grains (percent- } \\
\text { age of 100) }\end{array}$ & Default 15 , can be varied from $5-30$ \\
\hline misfit_threshold* & $\begin{array}{l}\text { Maximum allowable misfit between ellipse and } \\
\text { grain mask for filtering suspect grains (percent- } \\
\text { age of 100) }\end{array}$ & Default 30 , can be varied from $10-50$ \\
\hline min_size_threshold* & $\begin{array}{l}\text { Minimum area of grain, used to clean the mask } \\
\text { (pixels) }\end{array}$ & $\begin{array}{l}\text { Default } 10 \text { for } \sim 1 \mathrm{~mm} / \text { pixel imagery, } 40 \text { for }<0.8 \\
\mathrm{~mm} / \text { pixel }\end{array}$ \\
\hline first_nl_denoise* & Strength of first non-local means denoising & Default 5 , can be varied \pm 1 \\
\hline tophat_th* & $\begin{array}{l}\text { Upper percentile threshold to take from top-hat } \\
\text { filter for edge detection (fraction of } 1 \text { ) }\end{array}$ & Default 0.9 , can be varied from $0.8-0.95$ \\
\hline sobel_th* & $\begin{array}{l}\text { Upper percentile threshold to take from sobel fil- } \\
\text { ter for edge detection (fraction of } 1 \text { ) }\end{array}$ & Default 0.9 , can be varied from $0.8-0.95$ \\
\hline canny_sig* & Canny filtering sigma value for edge detection & Default 2 , can be varied from $1-2$ \\
\hline resize & Value to resize windows by (fraction of 1 ) & $\begin{array}{l}\text { Default } 0.8 \text {, can be varied from } 0.5-0.99 \text { if you want } \\
\text { a smaller }(0.5) \text { or larger }(0.99) \text { pop-up window }\end{array}$ \\
\hline
\end{tabular}

*Influence on results 


\section{S2. GSDs Separated by Site}

Figure S1 and S2 show the results of the KMS approach to grain sizing on a site-by-site basis before and following a 20-pixel lower truncation of the distribution. On the left columns are the initial runs of KMS PebbleCounts without any lower truncation, and on the right columns are the re-running with a 20-pixel truncation. Aside from three sites (S09, S10, and S24) the $p$-values

5 for the KS-tests without truncation were $<0.01$, indicating a robust rejection of the null hypothesis. Following truncation, all 12 sites have $p$-values $>0.1$, except $\mathrm{S} 16$, which has $p=0.09$.

Despite a high $p$-value, S24 demonstrates a stronger bias in the GSD towards coarser grains (up to $0.5 \psi$ discrepancy), as indicated by the high $A_{\text {diff }}$ value of -1.36 . Here, the KS-test pass is likely caused by the small sample size remaining after truncation ( $n=24)$, the least of any site. The poor performance of S24 was expected given the large size range with many sub$10 \mathrm{~cm}$ pebbles and a few large boulders, strong cast shadows from the large grains, and intra-granular edges on angular boulders with quartz veins (see Figure 9b in the main manuscript). Importantly, S24 is the only site not from a major river stem, but rather from a debris-flow fan draining a small tributary catchment in the Quebrada del Toro. The site S34 also had a high $A_{\text {diff }}=-2.11$. In this case, poor performance is due to significant blurriness of this image, and again a small sample size $(n=47)$. 


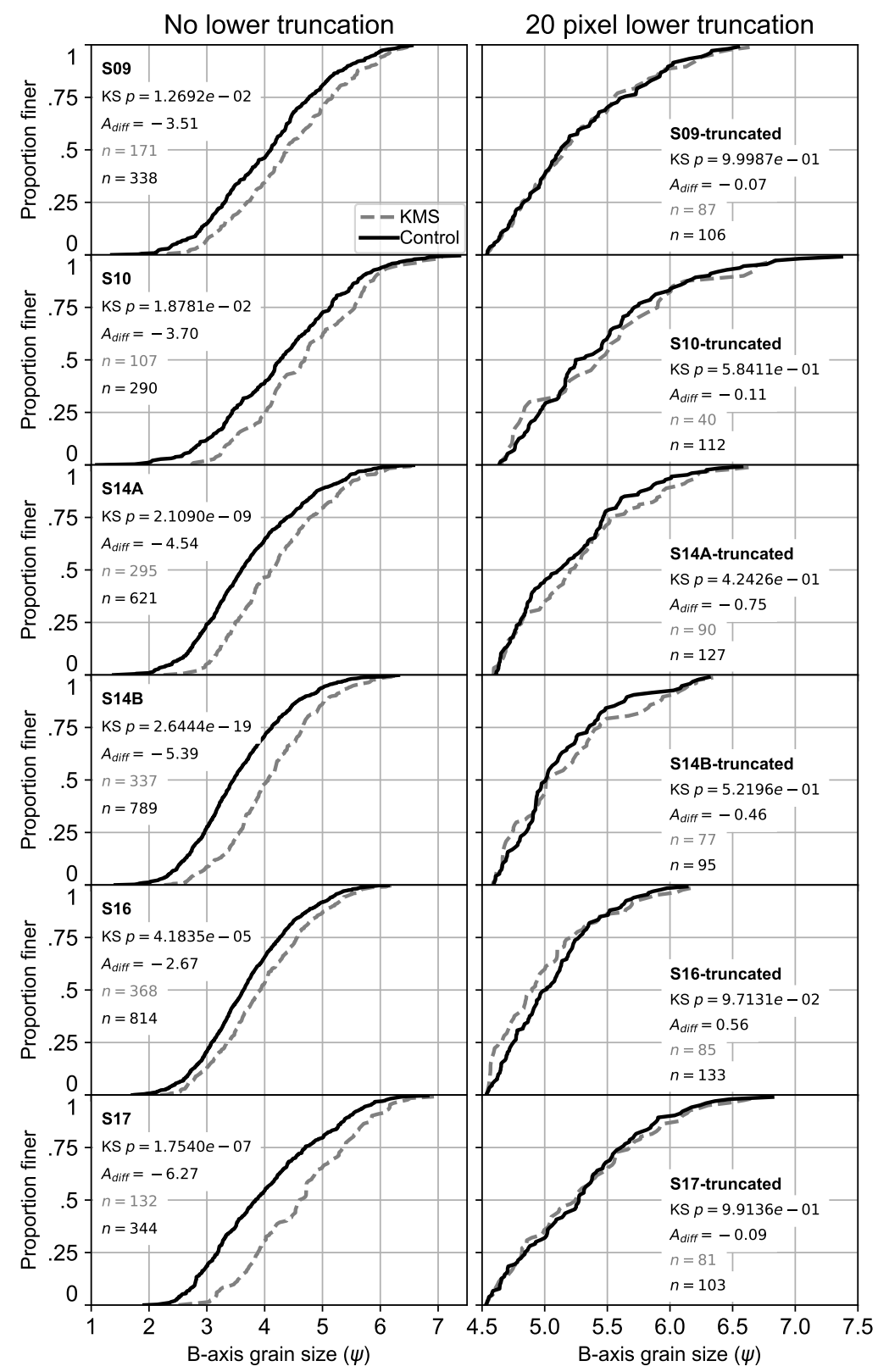

Figure S1. Results from KMS PebbleCounts on a site-by-site basis with the initial run in the left columns (no truncation) and the truncated (at 20-pixels) run in the right columns. The control data are given as a solid black line with the number of pebbles ( $n$ ) shown in black. KMS PebbleCounts results are in gray and dashed. The $p$-value results of a KS-test are also shown. $A_{\text {diff }}$ is the approximate integral between the curves. Note the reduction in $\mathrm{x}$-axis scale between the columns, where the right, truncated distributions are plotted on a narrower range to emphasize the remaining discrepancies. 


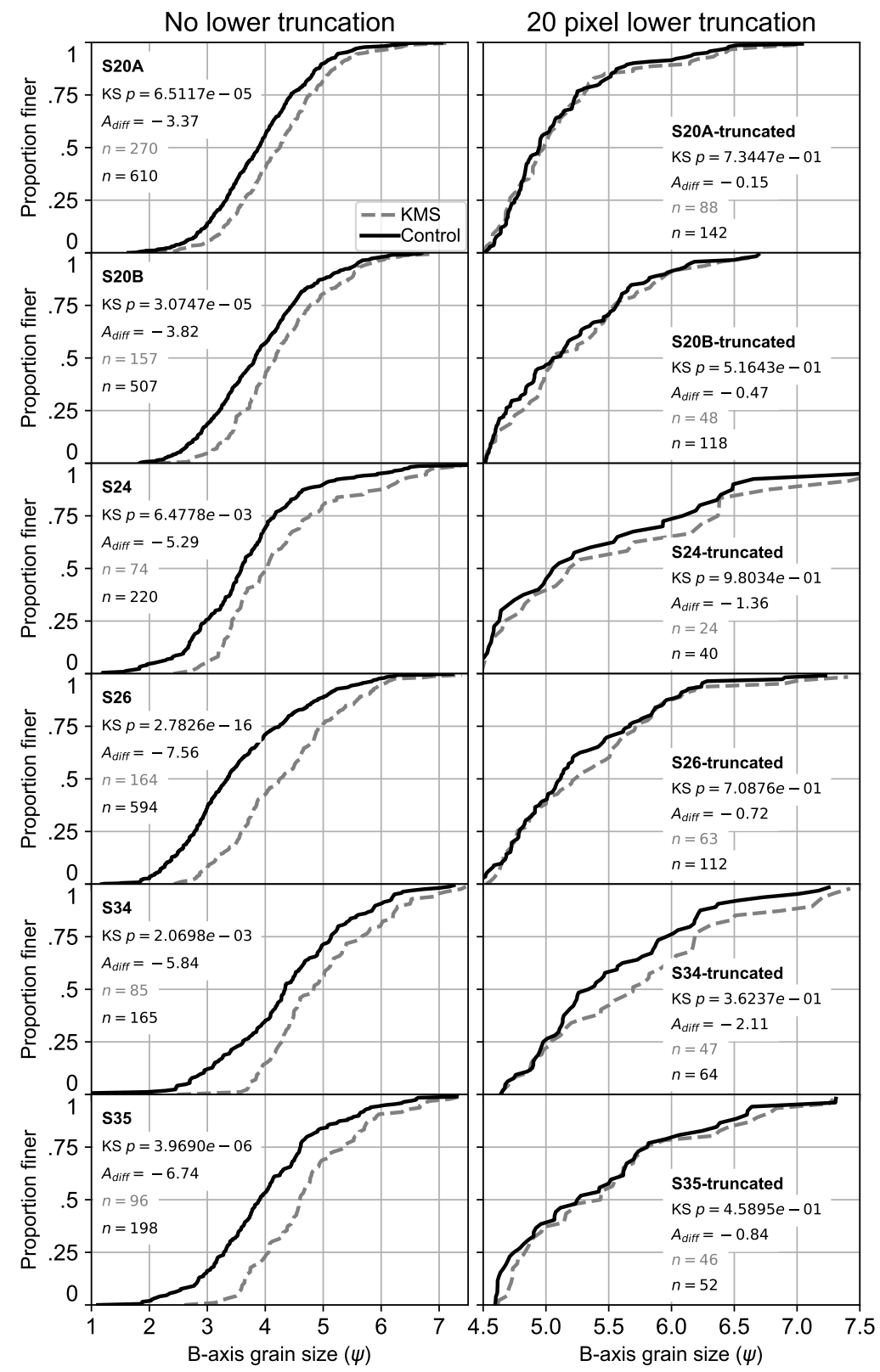

Figure S2. Results from KMS PebbleCounts on a site-by-site basis with the initial run in the left columns (no truncation) and the truncated (at 20-pixels) run in the right columns. The control data are given as a solid black line with the number of pebbles ( $n$ ) shown in black. KMS PebbleCounts results are in gray and dashed. The $p$-value results of a KS-test are also shown. $A_{\text {diff }}$ is the approximate integral between the curves. Note the reduction in $\mathrm{x}$-axis scale between the columns, where the right, truncated distributions are plotted on a narrower range to emphasize the remaining discrepancies. 

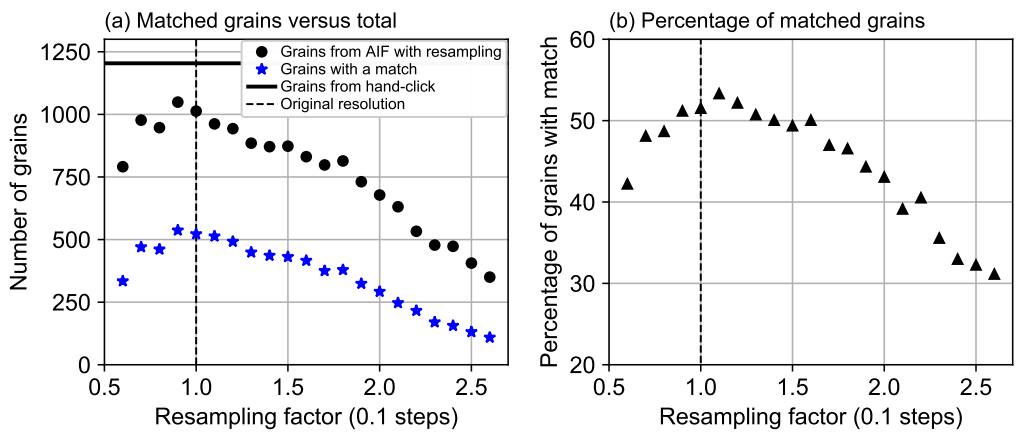

Figure S3. Matching grains found in each filtered mask versus the resampling factor (where 1 is the original image) for the $\sim 1.16 \mathrm{~mm} /$ pixel resolution images. Matches are defined as an AIF grain within 5 pixels of the hand-clicked line or the KMS grain centroid and with a $1 \mathrm{~cm}$ maximum b-axis difference between the AIF grain and the match.

\section{S3. Resampling and Parameter Selection in AIF Approach}

Figure S3 demonstrates the percentage of grains with a match found in the AIF approach (matches are defined as in main manuscript Figure 18) when increasing resampling from a factor of 0.6-2.6 by 0.1 steps using Lanczos resampling (Lanczos, 1950). As the resampling factor increases, there is progressive reduction in the number of found grains after filtering, therefore

5 we selected the original resolution (resampling factor of one). Figure S4 and Figure S5 demonstrate two cases where the resampling slightly improved the resulting GSD. Both images were of relatively low quality with significant blurring and the presence of many weak edges between grains of similar color.

We selected a maximum percent misfit between the ellipse and grain of $30 \%$ as the $90^{\text {th }}$ percentile of misfits for the KMS approach was $30 \%$. Furthermore, we allowed a maximum overlap between neighboring ellipses of $15 \%$, visually selected to minimize overlapping grain measurement and over-segmentation of discrete grains. For the higher resolution imagery it was necessary to use a lower sobel and top-hat threshold (0.85), since we consider all the edges at once in the AIF approach, rather than in a windowed subset as in the KMS approach, and many edges are not found when using the 0.9 threshold given the increased number of pixels under consideration. 


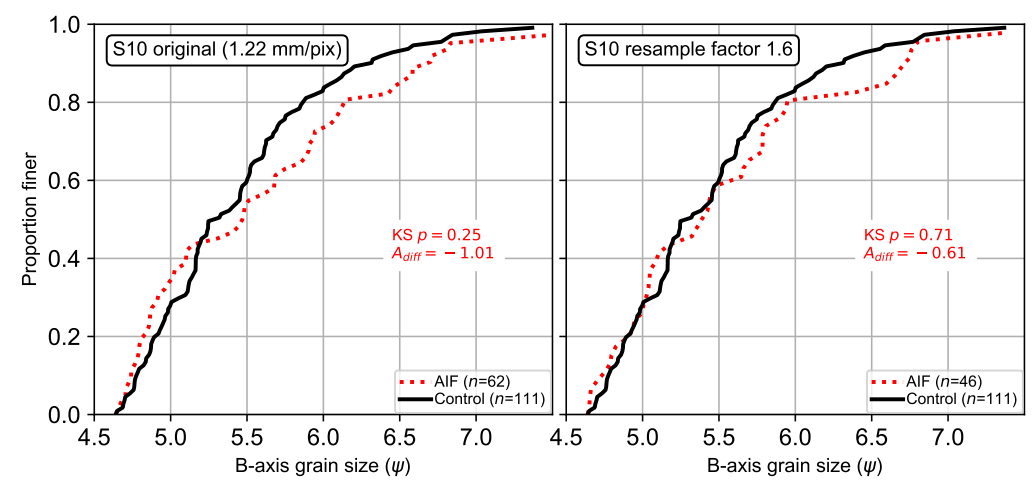

Figure S4. Slight improvement (increase in $p$ and decrease in $A_{\text {diff }}$ ) in result using a 1.6-times resampling factor prior to running the AIF algorithm for the difficult (somewhat blurry, weak edges) S10 orthoimage.

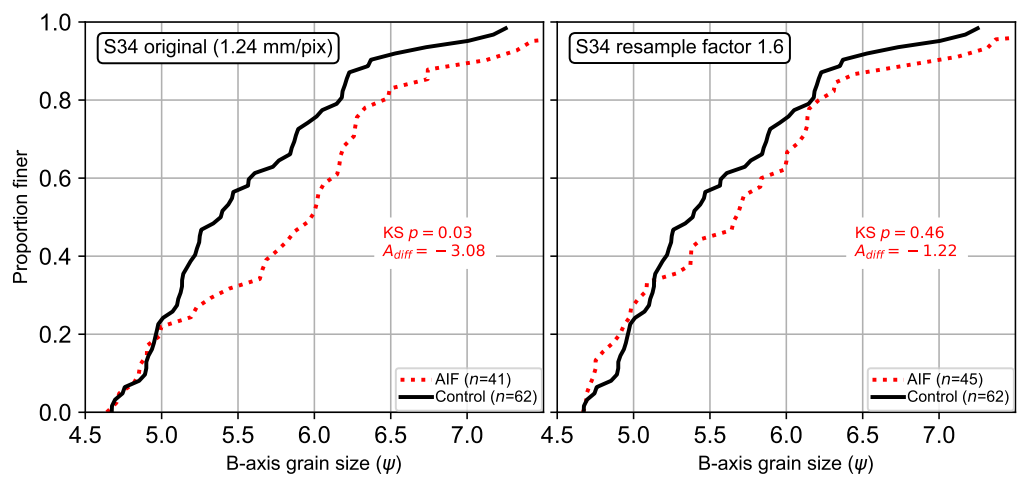

Figure S5. Slight improvement (increase in $p$ and decrease in $A_{\text {diff }}$ ) in result using a 1.6-times resampling factor prior to running the AIF algorithm for the difficult (very blurry, weak edges) S34 orthoimage. 


\section{References}

Lanczos, C.: An iteration method for the solution of the eigenvalue problem of linear differential and integral operators, J. Res. Natl. Bur. Stand. B, 45, 255-282, 1950. 